\title{
Characterization of RNA binding proteins required for receptor-mediated endocytosis in C. elegans
}

\author{
Margaret D. Holdeman ${ }^{1}$, Farida Daouda ${ }^{2}$, Heather A. Hundley, Ph.D. ${ }^{3}$ \\ ${ }^{1}$ Indiana University School of Medicine and Indiana Clinical and Translational Sciences \\ Institute, ${ }^{2}$ Louis Stokes Alliance for Minority Participation at Indiana University \\ Bloomington, ${ }^{3}$ Medical Sciences Program, Indiana University School of Medicine, \\ Bloomington, IN
}

Background and Hypothesis: Recently, a study conducted by the Hundley lab revealed that mutation of a specific RNA editing protein, $A D R 2$, in the model organism $C$. elegans increased receptor-mediated endocytosis of yolk protein vit-2 and oocyte maturation. Of interest to us was what additional RNA binding proteins were involved in the expression of vit-2. To this end, RNA interference (RNAi) was used to knock out relevant RNA binding proteins. We hypothesized we would find candidates that both enhanced and reduced the expression of vit-2.

Project Methods: Previously, adr-2(-) C. elegans strain expressing a yolk protein (vit-2) fused to GFP and a library of $E$. coli strains expressing RNAi directed to specific RNA binding proteins were engineered. vit-2:GFP reporter worms were grown on each RNAi bacterial strain. The COPAS Biosort, a large particle sorter which detects fluorescent intensity, was used to visualize and quantify vit-2 endocytosis.

Results: Our screen yielded ten candidate proteins. These proteins had various functions and were expressed in different tissues, most commonly the nervous and reproductive systems. Remarkably, proteins gld-1 and puf- 8 are negative regulators of oocyte fate determination and spermatogenesis respectively. Unlike the rest of our candidates, mutation of these two proteins did not have an observed effect on developmental progression but still caused significant increase in vit-2 expression.

Conclusion and Potential Impact: This screen successfully gave direction to future studies of $A D R-2$ involvement in yolk protein uptake, oocyte maturation, and embryonic development. Two of our identified targets seem to be involved in reproductive processes. The remainder may be affecting vit-2 expression by altering development or receptor-mediated endocytosis. Our goal in the immediate future will be to compare loss of these target proteins in wildtype worms to loss in adr-2(-) worms. This would add to what is known about ADARs and life span and possibly demonstrate a role for ADARs in germ cell maturation. 